\title{
A CIRCUMSTELLAR INTERACTION MODEL FOR SN 2002IC
}

\author{
T. Uenishi, ${ }^{1}$ T. Suzuki, ${ }^{1}$ K. Nomoto, ${ }^{1}$ and I. Hachisu ${ }^{2}$
}

SN 2002ic is a unique supernova which shows the typical spectral features of Type Ia supernovae (SNe Ia) near maximum light, but also apparent hydrogen features that have been absent in SNe Ia (Hamuy et al. 2003). We have calculated hydrodynamical models for the interaction between the SN Ia ejecta and the H-rich circumstellar medium (CSM) to reproduce the observed features of SN 2002ic. Based on our modeling, possible progenitor systems of SN 2002ic are discussed.

We calculated the interaction between the expanding ejecta and the CSM using spherical Lagrange hydrodynamical code (Suzuki \& Nomoto 1995). For the sur:ernova ejecta, we used the W7 model (Nomoto et al. 1984). For the CSM we assumed the density distribution:

$$
\rho=\rho_{0}\left(R / R_{0}\right)^{-n} \mathrm{~g} \mathrm{~cm}^{-3},
$$

where $R_{0}=1 \times 10^{15} \mathrm{~cm}$ is the radius where the ejecta and CSM interact and the density there is $\rho_{0}=1-25 \times 10^{-15} \mathrm{~g} \mathrm{~cm}^{-3}$.

Figure 1 shows the best fit model for the light curve of SN 2002ic, which has $\rho_{0}=2.5 \times 10^{-14} \mathrm{~g}$ $\mathrm{cm}^{-3}$ and $n=1.8$. On day 200 after the explosion, the mass of interacted CSM is $2-3 M_{\odot}$. The model with $n=2.0$ (steady mass loss) declines too fast to be compatible with the observation. Therefore, CSM around the SN was created by unsteady mass loss of the progenitor system. We note in Figure 2 that the velocity of the ejecta is $\sim 4000 \mathrm{~km} \mathrm{~s}^{-1}$ and too low for observed value $\left(\sim 10000 \mathrm{~km} \mathrm{~s}^{-1}\right)$.

To reproduce both the light curve and the observed velocity of SN 2002ic, we need asperical CSM. Suppose the CSM is aspherical consisting of a dense region and a thin region. The expanding ejecta interacting strongly with the dense region can produce high enough luminosity to explain the light curve. On the other hand, the ejecta interacting with the thin region can expand still fast enough to be consistent with the observed velocities.

\footnotetext{
${ }^{1}$ Department of Astronomy, School of Science, University of Tokyo 7-6-1 Hongo Bunkyo-ku, Tokyo, 113-0033, Japan.

${ }^{2}$ Department of Earth Science and Astronomy, College of Arts and Science, University of Tokyo, Meguro-ku Tokyo, 1538902, Japan.
}

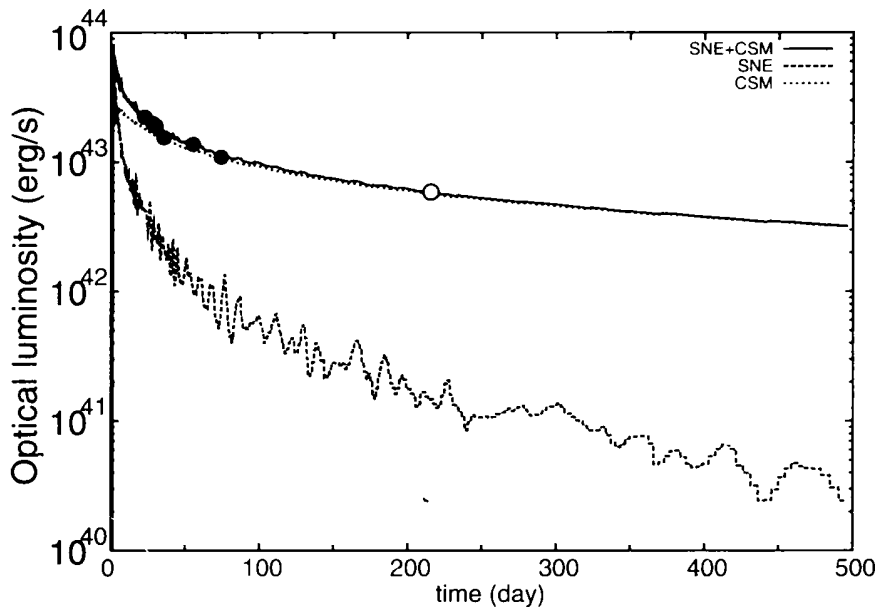

Fig. 1. Calculated light curve with observed data points (Subaru data point (Deng et al. 2003) is shown in open circle).

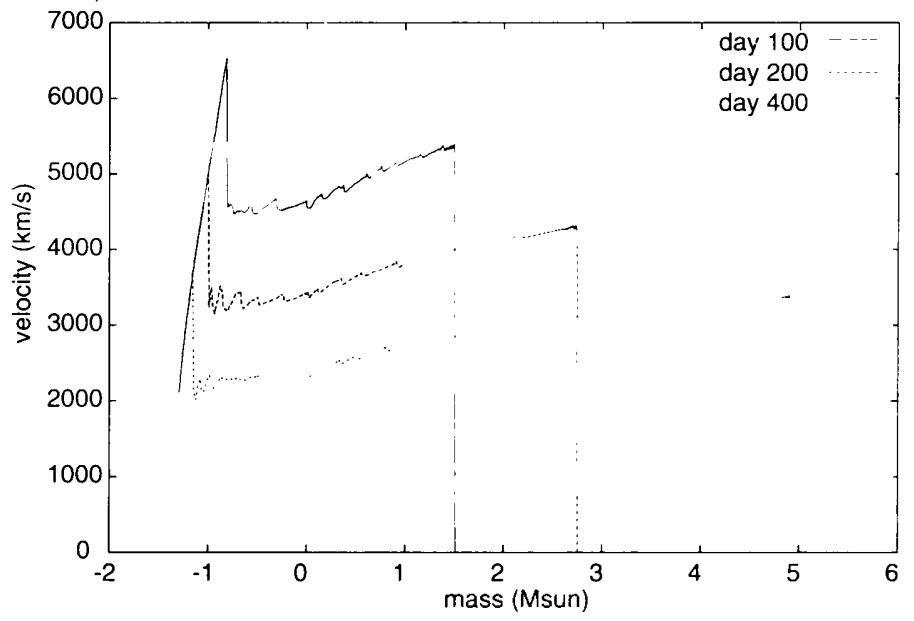

Fig. 2. Velocity profile.

There are two possible progenitor scenarios for SN 2002ic. One is the the explosion of the C+Ocore of the massive AGB star (SN I1/2), where the wind from the AGB star formed the ('S.I. The other is the explosion of the white dwarf in a close binary blowing wind to create the dense CSM.

\section{REFERENCES'S}

Deng, J., Kawabata, K. S.. Ohyama, Y., Xomoto, K.. Mazzali, P., Wang, L., Jeffery, D. J. \& Iye, M. 2003. ApJ, submitted (astro-ph/0311590)

Hamuy, M. et al. 2003, Nature, 42.4. 651

Nomoto, K., Thielemann, F-K. \& Yokoi, K. 19st. ApJ, 286,644

Suzuki, T. \& Nomoto, K. 1995, ApJ, 455, 6.5 